\title{
Management of haemophilia
}

Children with severe haemophilia A (factor VIII deficiency) or B (factor IX deficiency; Christmas disease) need frequent intravenous injections of blood products to control their bleeds. Present technology dictates that the products used are derived from human plasma, and this raw material and its derivatives contain many more ingredients than the clotting factors relevant to treatment. Some of these, for instance the virus of hepatitis $\mathbf{B}$, are well recognised as potentially harmful pathogens. Some, for instance the putative non-A, non-B viruses, cause over $90 \%$ of post-transfusion hepatitis Other agents may be implicated in the acquired immunodeficiency syndrome (AIDS). Immune complexes may play a role in this and in other transfusion related diseases. Given that human plasma carries these inherent dangers, are there safer alternatives? If not, how should the family with a severely affected child be counselled?

\section{Possible alternatives}

Animal factor VIII. The haemophilia factors are present in all vertebrate blood and 10 years ago preparations from cattle and pigs were available in the United Kingdom. They were used principally for treatment during and after surgery and for the management of complications, and they worked well. Both products, however, produced thrombocytopenia, and both induced a resistance in the recipient within 10 days of the start of treatment. The first problem, the result of platelet clumping, caused few difficulties. The second meant that the intermittent treatment of everyday bleeds was impossible. Bovine factor VIII is no longer available but a new porcine factor VIII (Hyate:C, Speywood Laboratories) is now licensed for use in this country. Hyate: $\mathrm{C}$ does not cause thrombocytopenia; and it has been used for more than 10 days in some patients. ${ }^{1}$ Resistance, however, is still encountered and, in its present form, it does not provide a safe and effective alternative to human products except in special circumstances-for instance in the management of some patients with high titre factor VIII antibodies.

Recombinant DNA products. Within the past year amino acid sequencing for both factors VIII and IX has been completed. Given that factor VIII is one of the largest molecules in the body and its active moiety one of the most labile, this is a remarkable achievement. It will almost certainly lead to artificial products for haemophilia management, perhaps solving the problems of viral, if not immune complex contamination; whether other side effects will emerge remains to be seen. It will be at least five years, however, before large scale clinical trials can be mounted with either product. In the meantime the technology should provide DNA probes of great value in the diagnosis and differentiation of haemophilic patients and haemophilia carriers, and for early, chorionic prenatal diagnosis.

Other haemostats. Bleeds in some sites or in more mildly affected children may sometimes respond to preparations other than those derived from blood; some of these are mentioned later. As the basic defect, however, in severe haemophilia is the complete absence of clotting activity of a specific factor, the only effective treatment is the replacement of that activity to provide a stable clot at the site of bleeding. This replacement must be systemic; unprotected local haemostats are simply washed away from all but the most minor of wounds.

\section{Counselling}

Advice to parents of affected children must be based on accurate knowledge of both the natural history of haemophilia and the effects and side effects of management. This statement may seem obvious but it is surprising how often doctors with little or no experience of haemophilia feel able to 'advise' families whose members may know far more about the disorder than they do. Even now one major paediatric text ${ }^{2}$ maintains that every possible step must be taken to prevent trauma, particularly in the early years of life. Young children should wear a protective helmet to guard the face and head.' Authoritative remarks like this are more likely to turn a potential handicap into a real one than any amount of haemophilic bleeding.

On average, the severely affected haemophiliac bleeds 35 times a year. Frequency changes with age being higher in children, partly because of the relative instability of joints and partly because of activity. A haemophilic child may bleed spontaneously two or three times a week, at any time. Two points need to be made about a child's pattern of bleeding. Firstly, it is a fallacy that protection by the avoidance of strenuous activity prevents bleeding. Experience both here and abroad has shown 
that only one accident in 15 might provoke a bleed in an otherwise healthy youngster. Secondly, the effects of haemophilia on the child and his family, especially when treatment is suboptimal, are far more profound than a glimpse of the physical pathology might suggest. It is vital to understand these aspects of haemophilia if the family is to be helped to cope in the most normal and beneficial way. It has been our policy for many years to encourage affected boys to participate in normal activities with their normal peers in normal schools. Outside hospital the haemophilia takes second place-only boxing, rugby football, and karate, those sports with a particular risk of head injury, being banned.

If haemarthroses are controlled effectively with early treatment and muscle development is good at adolescence, severe arthropathy in adult life is unlikely. Conversely, poor muscular development and poor coordination mean that major joints are ill supported, that bleeds are more frequent, and that when they occur they are harder to control.

\section{Home treatment}

The cornerstone of modern haemophilia management is home treatment. At most centres the parents of severely affected children have been taught how to give the relevant blood product intravenously by the time their child is 6 years old. At present most injections are given 'on demand' at the first evidence that a bleed has started. This evidence will not be visible-treatment given before the development of physical signs aborts bleeds with few if any sequelae, the child returning to school or play immediately. From the age of 3 years most severely affected boys have learnt to recognise the aura of a bleed, a 'fizzy' or 'funny' feeling that internal haemorrhage has begun. The other form of home treatment is prophylaxis, in which regular injections are given in an attempt to prevent bleeds. Evidence from Scandinavia suggests that prophylaxis started in the second year of life and continued into late adolescence will ablate haemophilic arthropathy. ${ }^{3}$ Although treatment may need to be given only once a week to achieve this, difficulties in arranging for very small children to have regular venepunctures and present worries about side effects have delayed the introduction of such regimens elsewhere. Instead most prophylaxis is initiated when a particular joint becomes the target of recurrent haemorrhage. If this happens alternate day injections plus the treatment of breakthrough bleeding often provide the answer, avoiding the need to use anti-inflammatory drugs or perform synovectomy.
The best products for home treatment are the lyophilised concentrates. They are easily stored and carried, have a long shelf life, and provide injections of known dosage in small volumes that can be prepared and given rapidly via syringe and butterfly needle. Their major disadvantage is that because the yield of clotting factor is inversely related to the purity of the final product, each batch of concentrate must be prepared from the plasma of multiple donors (up to 20000 ) and there is a much greater chance of viral or other pathogenic contamination than with single donor or small pool blood products. This is why wet cryoprecipitate, prepared from single volunteer donors, is still the preparation of choice for the treatment of small children with severe haemophilia A. With around 70 units of factor VIII per pack, an infant need be exposed to only three donors and a 4 year old to only eight donors per injection.

Because it is the source material for the lyophilised concentrates, the great majority of severely affected haemophilic patients cannot be treated solely with cryoprecipitate, whether wet or freeze dried, for life, and by school age most children are on concentrates. These may be prepared either by commercial companies using plasma from paid donors or by blood transfusion services relying on volunteer donations. Within the United Kingdom there is at present sufficient factor IX from volunteer sources to treat haemophilia $B$, but insufficient factor VIII for haemophilia $\mathrm{A}$, and $60 \%$ of these patients are treated with commercial concentrates which are readily available. On average 33000 units of factor VIII are required to treat one patient for one year in the United Kingdom, at a cost of about $£ 2600$.

\section{Some answers}

The principle side effect of haemophilia treatment remains hepatitis. Hepatitis $\mathrm{A}$ is not transmitted via blood but hepatitis $B$ is, and all children who will be exposed to blood products and are known not to have antibodies to hepatitis $B$ should be vaccinated with H-B-Vax (Morson; MSD). Vaccination in children under 10 years requires three intramuscular injections of $0.5 \mathrm{ml}$, the second after one and the third after six months. Although intramuscular injections are generally contraindicated in haemophilia, immunisation is safe provided pressure is kept over the site for five minutes after the procedure; it is extremely rare for factor replacement to be required because of haematoma formation.

Despite intensive research the aetiological agents of non-A, non-B hepatitis remain elusive. They are 
present in all blood products used for the treatment of haemostatic disorders, including the newer heat treated concentrates. Because non-A, non-B hepatitis is, however, more common after exposure to large donor pool concentrates there is a case for trying to maintain young (preschool) children on voluntarily donated cyroprecipitate. Despite recent publicity on the human $T$ cell lymphotrophic retrovirus type III the definitive cause of AIDS has yet to be established with certainty. ${ }^{4}$ If the syndrome is spread by blood transfusion (and the evidence that this is so remains sparse ${ }^{5}$ ) identification of the agent responsible should lead to appropriate screening tests. For the present, the same provisions as for hepatitis should apply to children, and parents should be reassured that, at least in the haemophilic population, AIDS is a relatively minor problem affecting only 1 in 1000 patients: only two adult cases of haemophilia and AIDS are known in the United Kingdom, and they were reported over a year ago. If the problems of non-A, non-B hepatitis and AIDS can be laid to rest there is a strong case for introducing prophylaxis, perhaps on a weekly basis, very early in childhood.

Another problem of relevance to the treatment of children has been the discovery of immune disorders in up to $10 \%$ of the haemophilic population. Thus we have seen idiopathic thrombocytopenic purpura and acquired haemolytic anaemia in otherwise well children in Newcastle. Non-specific lymphadenopathy has to date been confined to our adult patients. The emergence of these disorders in addition to the recurrent abnormalities of liver function make it obligatory that every child receiving blood products from whatever source is followed up rigorously at minimum intervals of six months.

Children with moderate or mild haemophilia and those with von Willebrand's disease should not receive large pool concentrates. In haemophilia $\mathrm{A}$ or von Willebrand's disease the factor VIII C (activity level) is raised sufficiently with desmopressin to cover most episodes of trauma or surgery; desmopressin does not work unless the patient is capable of producing some VIII C. Moderately or mildly affected haemophilia B children should be treated with fresh frozen plasma. Because response to factor IX in fresh frozen plasma is much poorer than that expected with factor VIII, any invasive procedure should be performed only under laboratory control. This rule applies to all children with haemostatic defects; seven per cent of haemophilia A and one per cent of haemophilia B patients develop antibodies (inhibitors) to the relevant factor and these must be excluded by specific testing and by measurement of response to transfusion before surgery or dental extractions. Children with the rarer recessively inherited disorders, for instance factor V deficiency, should receive fresh frozen plasma. The use of fresh whole blood is to be deprecated.

In all cases of open bleeding concomitant treatment with antifibrinolytics (Epsikapron, EACA, or Cyklokapron, AMCA) is indicated at least until wound healing is advanced. Antifibrinolytics are effective especially after dental extractions, but a firm clot has to be there in the first place for them to work. Antifibrinolytics should be used with care in conjunction with factor IX concentrates because of an increased risk of thromboembolism, and they are contraindicated if haematuria is present because renal tract obstruction with clot may occur.

The most important aspect of management remains the need for affected children and their families to have immediate access to centres providing comprehensive care. The aim of these haemophilia centres, which began in the United Kingdom, is to provide all the services required to treat bleeding effectively, together with advice and help with other problems based on experience with many patients. The requirements and location of these centres may be found in the Department of Health and Social Security health circular HC (76)4. The work of the centres is complemented by the lay Haemophilia Society (PO Box 9, 16 Trinity Street, London SE1) which families should be encouraged to join.

\footnotetext{
References

1 Kernoff PBA, Thomas ND, Lilley PA, Matthews KB, Goldman E, Tuddenham EGD. Clinical experience with polyelectrolyte - fractionated porcine factor VIII concentrate in the treatment of haemophiliacs with antibodies to factor VIII. Blood 1984;63:31-41.

2 Jolly H. Diseases of children. 4th ed. Oxford: Blackwell, 1981:528.

3 Nilsson IM, Hedner U, Ahlberg A. Haemophilia prophylaxis in Sweden. Acta Paediatr Scand 1976;65:129-35.

4 Anonymous. The cause of AIDS? [Editorial]. Lancet $1984 ; \mathbf{i}: 1053-4$

5 Bayer WL, Tegtmeier GE, Barbara JAJ. The significance of non- $A$, non-B hepatitis, cytomegalovirus and the acquired immune deficiency syndrome in transfusion practice. Clin Haematol 1984;13:253-69.
}

Peter Jones

Department of Haematology, Royal Victoria Infirmary, Newcastle upon Tyne 